\title{
Dem Andenken einer Forschergeneration.
}

Mythischer Nebel und die verwirrenden Irrlichter gegensätzlicher Wertungen umgeben bereits die Person KAARLE Krohns, des berühmtesten finnischen Folkloristen. Der Verfasser dieser Zeilen erinnert sich von seiner Schulzeit her des betagten Professors, wie er in einer Kulturveranstaltung sprach, sich enthusiasmierte und schliesslich in Weinen ausbrach - im übrigen bin ich von den durch starke Sympathie und Antipathie gefärbten Schilderungen abhängig, die Kaarle Krohns Schüler aufgefordert und unaufgefordert der jungen Generation geboten haben.

Den Baum kennt man an seinen Früchten, den Wissenschaftler an seinen Werken - und an seinen Schülern. Legen wir Kaarle Krohns eigene, auf einige zehn sich belaufende wissenschaftliche Werke in die Wagschale und als Gegengewicht dazu das schon auf etwa zweihundert Bände angewachsene Schaffen seiner Schüler - das Ergebnis zeigt, dass der Universitätslehrer Krohn nicht geringer gewesen ist als der Forscher Krohn.

Ich glaube, dass ein Überblick über die zwanzigköpfige Gruppe von Forschern, die in der 'Regentenzeit' Kaarle Krohns, nachdem sie in der finnischen und vergleichenden Folklore doktoriert hatten, den höchsten akademischen Grad erlangt haben, auch andere interessieren kann als nur die vier Doktoren der 'nachkrohnschen' Zeit. Die Geschichte des erwähnten Wissenschaftszweiges besteht eine lange Zeit aus der Geschichte dessen, was diese zwanzig getan und zu tun unterlassen haben.

o. Relander

$1863-1930$

Matti Waronen

$1861-1911$
29. 5. 1894. Kuvakielestä vanhemmassa suom. lyyrillisessä kansanrunoudessa (Über die Bildersprache in der älteren finn. lyrischen Volksdichtung). 27. 5. 1895 . Vainajainpalvelus muinaisilla suomalaisilla (Der Totenkult bei den alten Finnen).
Seminaroberlehrer und -direktor.

Seminaroberlehrer. 
I. R. Nimmi

$1869-1931$

K. I. Franssila $1869-1950$

Oskar Kallas $1868-1966$

Kaarlo Levón $1867-1936$

\section{Kartumen} 1874

J. W. Juvelius $1863-1927$

\section{Intti Iarne} 1867 -1925

o. J. Brummer $1878-1944$

\section{J. Iansikika} $1884-1947$

F. A. Hästesko (Heporauta) $1879-1946$

\section{Uno Ilolmbers}

(Ilarva) 1882-1949

A. A. Koskenjaakko 23. 5. 1913. Samanlaskututki1885

\section{Raf. Engelberg 1882 \\ Väinö Salminen $1880-1947$}

17. 12. 1898. Kalevalan kolioonpano I. Runokokous Vänämöisestä (Die Zusammensetzung des Kalevala I. Die I.iedersammlung über Väinämöinen).

29. 9. 1900. Kansanrunouden lutkimuksia 1. Iso tammi liitteincen (Untersuchungen zur Volksdichtung I. Die Grosse Eiche und die zugrehörigen Molive).

28. 5. 1901. Die Wiederholungsliader der estnischen Volkspoesic $I$.

6. 4. 1904. Tutkimuksia loitsurunojen alalla. Verensulkusanat ja raudan sanat. (Lintersuchungen auf dem Gebicte der Kauberlieder. Die Sprüche gegen den Blutfluss und über das Eisen).

16. 12. 1905. Kalevipoegin kokoonpano (Die Zusammenselzung des lialeviproeg).

7. 3. 1906. Länsi-Suomen käärmeen loitsut (Die westfinnischen Schlangenbeschwörun(ren).

23. 11. 190\%. Vergleichende Märchenforschungen.

17. 10. 1908. Uber die liannungsorte der finnischen Zauherlieder.

2'. 2. 1909. Über russische Zaluberformeln mit Berücksichtigung der Blut- und Ver-

26. I. 1910. Länsisuomalaiset tautien loitsut (Die westfinnischen Zaubersprüche gegen k'ankheiten).

16. 5. 1913. Dje Wassorgottheiten der finnisch-ugrischen Völker. muksia. Iaki, oikeus ja oikeudenkïynti suomal. sananlaskuissa. (Sprichwortforschungen. Gesetz, Recht und Rechtshandel in den finn. Sprichwörtern).

9. 5. 1914. Kalevalan sisällys ja rakenne (Gehalt und Struktur des Kalevala).

19. 5. 1917. Länsi-Inkerin häarunot. Synty- ja kehitysrenkungssegen. a. o. Professor 1918

-1930 , o. Prol.

$1930-1931$.

Schulrat bei der Obersten Schulbehörde.

Estnischer Gesandter in Helsinki und London.

Seminaroberlehrer.

Studiendirektor.

Studienrat.

a. o. Professor 1922 $-1925$

studienrat, Chefredakteur.

a. o. Professor 1927 $-1947$.

Studiendirektor des Normallyzeums.

Professor $\quad 1926-$ 1949.

Studiendirektor.

Organisations-

sekretär.

Professor 1933 1947. 
historiaa. (I)ie west-ingermanländischen Hochzeitslieder. Entstehungs- und Entwicklungsgeschichte).

A. V. Rantasalo $1881-$

Ilmari Manninen $189 / 1935$

Marti Haavio 1899

Elsa EnäjärviHaavio 1901 - 1951
19. 12. 1919. Vorbereitungen zum Säen und Pflanzen im Volksaberglauben der Finnen und Esten mit entsprechenden Gebräuchen der Germanen verglichen.

2. 12. 1922. Die dämonistischen Krankheiten im finnischen Volksaberglauben.

25. 9. 1929. Kettenmärchen- Professor 1950studien I.

27. 5. 1932. The Game of Rich and Poor. A comparative study in traditional singing games.
Dozent, studienrat.

Dozent, Museumsdirektor.

Dozent.

Es verlohnt sich zu beachten, dass von den erwähnten zwanzig Forschern acht Altersgenossen Kaarle Krohns sind und zu derselben in den sechziger Jahren des 19. Jahrhunderts geborenen Generation gehören wie zahlreiche glanzvolle Namen der neuesten Kultur- und politischen Geschichte Finnlands: Sibelius, Gallen-Kallela, Halonen, Wikström, die Gebrüder Järnefelt, Aho, Setälä, Westermarck, Ståhlberg, Svinhufvud, Mannerheim. Die zweite bedentsame Gruppe bilden die in den Jahren 1878-1885 Geborenen, ebenfalls an Zahl acht. Die folgenden 24 Jahrgänge haben alles in allem nur drei Doktoren hervorgebracht. I)as Interesse fiir die Folklore hat offenbar seinen eigenen Rhythmus; für die grosse Aufschwungsperiode zu Beginn des Jahrhunderts lassen sich Entsprechungen auf dem Gebiete des künstlerischen Schaffens zeigen (Sibelius', Gallen-Kallelas und Leinos Meisterwerke mit Kalevalamotiven).

Es scheint so, dass die frühesten Doktoranden ihre Themen selbst gewählt oder sie vielleicht schon von Julius Krohn, dem 1888 durch einen Unglücksfall gestorbenen Bahnbrecher auf diesem Wissenschaftsgebiete und Vater Kaarle Krohns bekommen haben. Zu Beginn des Jahrhunderts scheint Kaarle Krohn die Aufhellung eines Spezialgebietes - der Zauberspriuche - geplant zu haben unter gleichzeitiger Benutzung einer Reihe von Schülerarbeiten auf dieselbe Weise, wie er die Seminar- und Examensarbeiten zielbewusst danach ausrichtete, die Klärung bald des einen, bald des anderen Spezialgebietes herbeizuführen. Es muss bemerkt werden, dass nur einer von den fünf Forschern, die die Zaubersprüche untersuchten, seine wissenschaftliche Tätigkeit nach Erledigung der Dissertation fortgesetzt hat. Seit 1910 ist 
eine neue Tendenz in der Themenwahl zu sehen: Die Mythologie, die Magie, die Hochzeitslieder, die sprichwörter, die Iärchen und spiele bekommen jedes seinen eigenen Bearbeiter. Vom Standpunkt des wissenschaftlichen Nachwuchses und des eigenen 'Wohlbefindens' der Forscher' ist eine solche Arbeitsteilung offenbar vorteilhafter gewesen als die vorherige. (Offensichtlich ist ferner, dass das umfangreiche internationale Vergleichsmaterial die Forscher mehr in 'Schwung' gebracht hat als das eng nationale (Aarne, Mansikka, Harva, Elsa und Martti Haavio).

Etwa die IIälfte der Wissenschaftler, die Kaarle Krohn als Lehrer hatten, haben ihre wissenschaftliche Arbeit auch noch nach ihrer I issertation fortgesetzt; es dürfte kein Zweifel darüber bestehen, dass sich unter denen, die sie fortsetzten, die meisten echten Wissensehaftlerbegabungen jener zwanzig Doktoren befanden. Auf Verlustkonto ist wenigstens zu setzen, dass Ilatti Waronen und Oskar Kallas sich von der Wissenschaft anderen Berufen zuwandten. Am mächtigsten war die Expansion des von Kaarle Krohn geführten Wissenschaftsfaches in der zweiten Hälfte der zwanziger Jahre dieses Jahrhunderts, wo faktisch fünf Professoren und zwei Dozenten sich mit finnischer und vergleichender Folklore beschäftigten: Krohn selbst mit seinen synthetischen Zusammenfassungen "Die folkloristische Arbeitsmethode» und "Kalevalastudien I-VI», Setälä mit dem Monumentalwerk „Das Rätsel vom Sampo» und Harva mit den Vorarbeiten zu dem Buch „Die religiösen Vorstellungen der altaischen Völker», Niemi und Mansikka mit ihrem litauischen Material, Salminen und Rantasalo mit den Problemen der ingermanländischen Lieder, dazu Koskenjaakko mit Sprichwörtern und der von Antti Aarne geschulte junge Magister Martti Haavio mit den Kettenmärchen.

Es erhebt sich die Frage: Woher kam dann das tiefe Wellental auf diesem Wissenschaftsgebiete in den dreissiger und in der ersten Hälfte der vierziger Jahre dieses Jahrhunderts? War Kaarle Krohn eine Rieseneiche, die zwar einen dichten Nachwuchs säte, zugleich aber das Wachstum der jungen Schösslinge zu voller Höhe hinderte? Man weiss, wie energisch der warmherzige Führer der Schule den jungen Forschern sowohl die wirtschaftlichen als auch die methodischen Schwierigkeiten aus dem Wege räumte. Tatsache ist jedoch, dass von Krohns älteren Schülern nur Uno Harva, der Turkuer Professor, als Forscher eine Lebensarbeit geleistet hat, die sich mit Krohns eigener vergleichen lässt. Väinö Salminen und V. J. Mansikka, deren Schultern in den dreissiger Jahren 
des 19. Jahrhmnderts Kaarle Krolıns verpflichtendes Erbe aufgebürdet wurde, gehörten zweifellos seinerzeit zu den selbständigsten und betriebsamsten Forschern der 'Schule'; aber das schwergewicht ihrer wissenschaftlichen Lebensarbeit liegt deutlich in der Zeit vor ihrer Professur. Es ist menschlich verständlich, dass die wissenschaftliche Forschung -- die eigene und die andicrer - eine mehr und mehr periphere stellung im Interessenbereich dieser in ihrer Jugend begeistert und aufopferungsvoll arbeitenden, dann überdrüssig gewordenen und enttäuschten Beanten bekam. I)je Katastrophe von 1946-1947 - die Zurückweisung zweier I Iissertationen, die schon die Druckerlaubnis erhalten hatten bildete den tragisch-logischen schlusspunkt dieses Entwicklungsverlaufs. Später, als die Verantwortung auf die jüngsten Schüler Kaarle Krohns übergegangen war, hat sirll die Anzahl der Studierenden der Volkspoesie in kurzer theit vervielfacht und die Forschungsarbeit auf diesem Gebiete ist kräftig aufgeblüht; wemn man dies gesehen hat, kann man nicht umhin, der vergangenen dürftigen anderthalb Jahrzehnte mit einiger Wehmut zu gedenken.

Dieser Nekrolog auf die Generation von Schülern Kaarle Krohns dürfte nicht allzu verfü̈ht sein: fünfzehn von den zwanzig sind schon dahingeschieden und von den fünf iibriggebliebenen drei im Ruhestand. Ich habe in diesen zwanzig vergilbten Abhandlungen von sehr verschiedener Art gebättert; ich habe die zum Wohle von Universität, Schule oder Gesellschaft geleistete Lebensarbeit der Zwanzig mitcinander verglichen; die gewünschten Gedenkworte auf zwei gestorbene Wissenschaftler sind zu einer Art kollektiver Ehrenbezengung für eine ganze Forschergeneration geworden: fïr die Generation der Schüler Kaarle Krohns. Ein jedes IItgrlied dieser Gruppe hätte zweifellos eine Einzelwürdignung verdient: aber rom Hintergrund der ganzen Gruppe hel,t sich sichthar ab die Person des grossen Lehrers, Inspirators und Organisators, die noch nach Jahrzehnten Früchte tragende erzieherische Leistung Kanle Krohns.

MatTi Kites. 